UDC 517.54

Rajbala, Jugal K. Prajapat

\title{
HARMONIC MAPPINGS WITH THE FIXED ANALYTIC PART
}

\begin{abstract}
In this article, we consider a class of sense-preserving harmonic mappings whose analytic part is convex in one direction. We prove that functions in this class are close-to-convex for certain values of parameters. Further, we obtain bounds on preSchwarzian derivatives and bounds on the Bloch's constant. Finally, we obtain coefficient bounds, growth and distortion results.
\end{abstract}

Key words: Univalent harmonic mappings, functions convex in one direction, pre-Schwarzian derivative, Bloch's constant, coefficient bound, growth and distortion results

2010 Mathematical Subject Classification: 30C45, 30C80

1. Introduction. Let $\mathcal{H}$ denote the class of harmonic mappings $f=h+\bar{g}$ in the open unit disk $\mathbb{D}=\{z \in \mathbb{C}:|z|<1\}$, normalized by $h(0)=0=h^{\prime}(0)-1$ and $g(0)=0$. The function $f=h+\bar{g} \in \mathcal{H}$ has the form

$$
h(z)=z+\sum_{n=2}^{\infty} a_{n} z^{n} \quad \text { and } \quad g(z)=\sum_{n=1}^{\infty} b_{n} z^{n} \quad(z \in \mathbb{D}) .
$$

The harmonic mapping $f$ is locally univalent, if and only if its Jacobian $J_{f}(z)=\left|h^{\prime}(z)\right|^{2}-\left|g^{\prime}(z)\right|^{2}$ does not vanish, and $f$ is sense-preserving if the Jacobian is positive. The analytic function $w=g^{\prime} / h^{\prime}$ is called the second complex dilatation of $f$, hence a sense-preserving harmonic mapping has the property $|w|<1$ in $\mathbb{D}$. We denote by $\mathcal{S}_{\mathcal{H}}$ the subclass $\mathcal{H}$ consisting of sense-preserving univalent harmonic mappings in $\mathbb{D}$. Let $\mathcal{S}_{\mathcal{H}}^{0}=\{f=h+$ $\left.+\bar{g} \in \mathcal{S}_{\mathcal{H}}: g^{\prime}(0)=0\right\}$. It is well known that the class $\mathcal{S}_{\mathcal{H}}^{0}$ is compact and normal, whereas the class $\mathcal{S}_{\mathcal{H}}$ is normal but not compact. The harmonic

(c) Petrozavodsk State University, 2021 
mappings has been extensively studied in the recent years; for a detailed discussion, we refer to [10], [15-17], [19] and the references therein.

A domain $\Delta$ is close-to-convex, if the complement of $\Delta$ can be written as the union of non-intersecting half-lines. A harmonic mapping $f$ is closeto-convex in $\mathbb{D}$, if $f(\mathbb{D})$ is a close-to-convex. In [6], Cluine and Sheil-Small proved the following result, which gives a sufficient condition for harmonic mappings to be close-to-convex in $\mathbb{D}$ :

Lemma A. If $h, g$ are analytic in $\mathbb{D}$ with $\left|h^{\prime}(0)\right|>\left|g^{\prime}(0)\right|$ and $h+\epsilon g$ is close-to-convex for each $\epsilon(|\epsilon|=1)$, then $f=h+\bar{g}$ is close-to-convex in $\mathbb{D}$.

Let $\mathcal{A}$ denote the class of analytic functions $h$ in $\mathbb{D}$ with the normalization $h(0)=0=h^{\prime}(0)-1$, and $\mathcal{S}$ denote the subclass of $\mathcal{A}$ consisting of univalent functions in $\mathbb{D}$. An analytic function $h$ is convex in the direction $\phi$, if every line parallel to the line through 0 and $e^{i \phi}$ has a connected or empty intersection with $h(\mathbb{D})$. A locally univalent function $h \in \mathcal{A}$ is in $\mathcal{S}^{*}$ if and only if $\operatorname{Re}\left(z h^{\prime}(z) / h(z)\right)>0$ for each $z \in \mathbb{D}$. Functions in $\mathcal{S}^{*}$ are referred to as the star-like functions (with respect to $h(0)=0$ ) in $\mathbb{D}$. A function $h \in \mathcal{A}$ is said to be close-to-convex in $\mathbb{D}$, if $h(\mathbb{D})$ is a close-to-convex, equivalently, if there exists a function $g \in \mathcal{S}^{*}$, such that $\operatorname{Re}\left(z h^{\prime}(z) / g(z)\right)>0$ for $z \in \mathbb{D}$.

Let $\mathcal{B}_{\alpha}(\alpha \in[0,1))$ denote the class of analytic functions $w$ in $\mathbb{D}$ satisfying $|w(0)|=\alpha$ and $|w(z)|<1$ for each $z \in \mathbb{D}$. If $f$ and $g$ are two analytic functions in $\mathbb{D}$, then we say that $f$ is subordinated to $g$, written as $f \prec g$, if there exists a function $w \in \mathcal{B}_{0}$ such that $f(z)=g(w(z))$. If $w \in \mathcal{B}_{\alpha}$, where

$$
w(z)=c_{0}+c_{1} z+c_{2} z^{2}+\ldots, \quad\left|c_{0}\right|=\alpha, z \in \mathbb{D},
$$

then we have

$$
\left|c_{n}\right| \leqslant 1-\left|c_{0}\right|^{2}, \quad\left|w^{\prime}(z)\right| \leqslant \frac{1-|w(z)|^{2}}{1-|z|^{2}}, \quad z \in \mathbb{D},
$$

(see e.g. [1, p. 30, 53]). In [11], Ma and Minda proved the following result for subordination:

Lemma B. Let $\psi$ be a starlike function, such that $\psi(0)=1$, and suppose that $g \in \mathcal{A}$ satisfies the equation

$$
1+\frac{z g^{\prime \prime}(z)}{g^{\prime}(z)}=\psi(z), \quad z \in \mathbb{D}
$$


Then, for $h \in \mathcal{A}$, the condition

$$
1+\frac{z h^{\prime \prime}(z)}{h^{\prime}(z)} \prec \psi(z), \quad z \in \mathbb{D},
$$

implies that $h^{\prime}(z) \prec g^{\prime}(z)$.

A locally univalent function $h \in \mathcal{A}$ belongs to the class $\mathcal{G}(\beta)$ for $\beta \in(2 / 3,1]$ if it satisfies the condition

$$
\operatorname{Re}\left(1+\frac{z h^{\prime \prime}(z)}{h^{\prime}(z)}\right)<\frac{3}{2} \beta, \quad z \in \mathbb{D} .
$$

In [20], Umezawa studied the class $\mathcal{G}(\beta)$ and proved that functions in $\mathcal{G}(\beta)$ are convex in one direction. Also, Ozaki [14] proved that functions in the class $\mathcal{G}(1) \equiv \mathcal{G}$ are univalent in $\mathbb{D}$. Further, Ponnusamy and Vasudevarao [18] proved that functions in $\mathcal{G}$ are starlike in $\mathbb{D}$.

The analytic part $h$ of a harmonic mapping $f=h+\bar{g}$ plays a crucial role in shaping the geometric properties of $f$ (see [6, Theorem 5.17]. Motivated by this idea, we define a new class $G_{\mathcal{H}}(\alpha, \beta)$ of harmonic mappings $f \in \mathcal{H}$ as follows.

Definition 1. Suppose that $w \in \mathcal{B}_{\alpha}$. Let $G_{\mathcal{H}}(\alpha, \beta)$ denote the class of harmonic mappings $f=h+\bar{g} \in \mathcal{H}$, such that $h \in \mathcal{G}(\beta)$ for each $\beta \in(2 / 3,1]$, and $g$ is given by $g^{\prime}(z)=w(z) h^{\prime}(z)$ for all $z \in \mathbb{D}$.

The pre-Schwarzian derivative $P_{h}$ of a locally univalent analytic function $h$ in $\mathbb{D}$ is defined by $P_{h}(z)=h^{\prime \prime}(z) / h^{\prime}(z)$ and the pre-Schwarzian norm of $f$ is defined by

$$
\left\|P_{h}(z)\right\|=\sup _{z \in \mathbb{D}}\left(1-|z|^{2}\right)\left|P_{h}(z)\right| .
$$

It is well known that $\left\|P_{h}\right\|<\infty$ if and only if $h$ is uniformly locally univalent, or equivalently, $h$ is univalent on each hyperbolic disk in $\mathbb{D}$ with fixed radius (see [21]). The pre-Schwarzian derivative and pre-Schwarzian norm of locally univalent analytic functions are widely used tools in the study of geometric properties of such functions. For instance, these can be used to get conditions for global univalence, or to obtain certain geometric conditions on the range of $h$. More specifically, if $f$ is univalent in $\mathbb{D}$, then

$$
\left|P_{h}(z)\right| \leqslant 6 /\left(1-|z|^{2}\right) \quad(z \in \mathbb{D}),
$$


and the bound 6 is sharp. Conversely, if $h$ is locally univalent analytic function in $\mathbb{D}$ and

$$
\sup _{z \in \mathbb{D}}\left|z P_{h}(z)\right|\left(1-|z|^{2}\right) \leqslant 1
$$

then $f$ is univalent in $\mathbb{D}$ (see [2]).

The notion of pre-Schwarzian derivative was generalized by Chuaqui et $a l[5]$ for locally univalent harmonic mapping $f=h+\bar{g}$ in $\mathbb{D}$ with dilation $w=q^{2}$, where $q$ is some analytic function. The pre-Schwarzian derivative for a locally univalent harmonic mapping $f=h+\bar{g}$ is given by

$$
P_{f}(z)=2 \frac{\partial}{\partial z}(\log \lambda)=\frac{h^{\prime \prime}(z)}{h^{\prime}(z)}+\frac{2 q^{\prime}(z) \overline{q(z)}}{1+|q(z)|^{2}}, \quad z \in \mathbb{D},
$$

where $\lambda=\left|h^{\prime}\right|+\left|g^{\prime}\right|$ and dilatation $w=g^{\prime} / h^{\prime}=q^{2}$ for some analytic function $q$. In the case when $f$ is analytic, (3) agrees with the pre-Schwarzian derivative for analytic functions. The pre-Schwarzian derivative $P_{f}$ has one disadvantage, that arises from the fact that dilatation $w$ is required to fulfil an extra condition, i.e. $w$ is equal to the square of an analytic function.

Another definition of pre-Schwarzian derivative $\mathcal{P}_{f}$ for sense-preserving harmonic mapping $f$ in a simply connected domain $\Omega \subset \mathbb{C}$ was given by Hernández and Martín [8] by $\mathcal{P}_{f}=\left(\log \left(J_{f}\right)\right)_{z}$, where $J_{f}$ is the Jacobian of $f$. If $f=h+\bar{g}$, then

$$
\mathcal{P}_{f}(z)=\frac{h^{\prime \prime}(z)}{h^{\prime}(z)}-\frac{\overline{w(z)} w^{\prime}(z)}{1-|w(z)|^{2}}, \quad z \in \Omega .
$$

Also, Hernández and Martin [8, Theorem 8] proved that if $f=h+\bar{g}$ is sense-preserving in $\mathbb{D}$ and

$$
\left|z \mathcal{P}_{f}(z)\right|+\frac{\left|z w^{\prime}(z)\right|}{1-|w(z)|^{2}} \leqslant \frac{1}{1-|z|^{2}}, \quad z \in \mathbb{D},
$$

then $f$ is univalent in $\mathbb{D}$. The constant 1 is sharp.

The classical Bloch theorem asserts that there always exists a positive constant $b$, such that for any holomorphic mapping $f$ of the unit disk $\mathbb{D}$ with $f^{\prime}(0)=1$, the image $f(\mathbb{D})$ contains a Schlicht disk of radius $b$. By Schlicht disk we mean a disk that is the univalent image of some region in $\mathbb{D}$. The Bloch constant is defined by supremum of such radius $b$. 
Chen et al [3] estimated the Bloch constant for harmonic mappings. A harmonic function $f$ is called harmonic Bloch mapping if and only if

$$
\mathcal{B}_{f}=\sup _{z, w \in \mathbb{D}, z \neq w} \frac{|f(z)-f(w)|}{\mathcal{D}(z, w)}<+\infty,
$$

where

$$
\mathcal{D}(z, w)=\operatorname{arctanh}\left|\frac{z-w}{1-\bar{z} w}\right|
$$

denotes the hyperbolic distance between $z$ and $w$ in $\mathbb{D}$, and $\mathcal{B}_{f}$ is called the Bloch's constant of $f$. In [7], Colonna proved that

$$
\mathcal{B}_{f}=\sup _{z \in \mathbb{D}}\left(1-|z|^{2}\right) \Lambda_{f}
$$

where

$$
\Lambda_{f}=\max _{0 \leqslant \theta \leqslant 2 \pi}\left|f_{z}(z)-e^{-2 i \theta} f_{\bar{z}}(z)\right|=\left|f_{z}(z)\right|+\left|f_{\bar{z}}(z)\right| .
$$

Moreover, the set of all harmonic Bloch mappings forms a complex Banach space with norm

$$
\|f\|=|f(0)|+\sup _{z \in \mathbb{D}}\left(1-|z|^{2}\right) \Lambda_{f}(z) .
$$

Recently, many authors have studied Bloch's constant for harmonic mappings (see [4], [9]).

For $h \in \mathcal{A}$ of the form (1), the classical Fekete-Szegö functional $F_{\lambda}=a_{3}-\lambda a_{2}^{2}$ plays an important role in function theory. The problem of maximizing the absolute value of the functional $F_{\lambda}$ is called the FeketeSzegö problem. We will obtain bounds on Fekete-Szegö functional for the class $G_{\mathcal{H}}(\alpha, \beta)$. For this, we need the following Lemma (see [13, Th. 1 and Th. 2]):

Lemma C. Let $h \in \mathcal{G}(\beta)$ for some $\beta \in(2 / 3,1]$ have the form (1). Then

a) $\left|a_{n}\right| \leqslant \frac{3 \beta-2}{n(n-1)}$ for all $n \geqslant 2$. The equality is attained for the function $h_{n}$, such that $h_{n}^{\prime}(z)=\left(1-z^{n-1}\right)^{(3 \beta-2) /(n-1)}, n \geqslant 2$.

b) $\left|a_{3}-\lambda a_{2}^{2}\right| \leqslant\left\{\begin{array}{l}\frac{3 \beta-2}{2}\left|1-\beta-\frac{3 \beta-2}{2} \lambda\right| \text { for }\left|\lambda-\frac{2(1-\beta)}{3 \beta-2}\right| \geqslant \frac{2}{3(3 \beta-2)} \\ \frac{3 \beta-2}{6} \text { for }\left|\lambda-\frac{2(1-\beta)}{3 \beta-2}\right|<\frac{2}{3(3 \beta-2)} .\end{array}\right.$

The equality is attained for the function $h$, such that

$$
h^{\prime}(z)=\left(1-z e^{i \theta}\right)^{3 \beta-2}, \quad \theta \in[0,2 \pi] .
$$


In this paper, we prove that the functions in the class $G_{\mathcal{H}}(\alpha, \beta)$ are close-to-convex for certain values of $\alpha$ and $\beta$. Further, we obtain bounds on pre-Schwarzian derivatives and bounds on the Bloch constant for the class $G_{\mathcal{H}}(\alpha, \beta)$. Finally, we obtain the coefficient estimates, growth and distortion results for the functions belonging to the class $G_{\mathcal{H}}(\alpha, \beta)$.

2. Main Results. The first result provides the close-to-convexity criteria for a harmonic mapping in the class $G_{\mathcal{H}}(\alpha, \beta)$.

Theorem 1. Let $\alpha \in[0,1)$ and $\beta \in\{1 / 3,1\}$. If $f=h+\bar{g} \in G_{\mathcal{H}}(\alpha, \beta)$ and

$$
W(z)=z^{\frac{3 \beta-1}{2}}\left(1+\epsilon \frac{g^{\prime}(z)}{h^{\prime}(z)}\right)
$$

is starlike in $\mathbb{D}$ for each $\epsilon(|\epsilon|=1)$, then $f$ is close-to-convex and, hence, univalent in $\mathbb{D}$.

Proof. Let $f=h+\bar{g} \in G_{\mathcal{H}}(\alpha, \beta)$, then $h \in \mathcal{G}(\beta)$. By definition,

$$
1+\frac{z h^{\prime \prime}(z)}{h^{\prime}(z)} \prec \frac{1+(1-3 \beta) z}{1-z}, \quad z \in \mathbb{D} .
$$

In view of the Herglotz representation for analytic functions, it follows that

$$
\frac{z h^{\prime \prime}(z)}{h^{\prime}(z)}=-(3 \beta-2) \int_{\partial \mathbb{D}} \frac{\bar{x} z}{1-\bar{x} z} d \mu(x), \quad z \in \mathbb{D},
$$

where $\mu$ is a probability measure on $\partial \mathbb{D}$, such that $\int_{\partial \mathbb{D}} d \mu(x)=1$. Therefore,

$$
\log h^{\prime}(z)=(3 \beta-2) \int_{\partial \mathbb{D}} \log (1-\bar{x} z) d \mu(x), \quad z \in \mathbb{D},
$$

and, thus, we have a sequence of functions $\left\{h_{n}(z)\right\}$, analytic in $\mathbb{D}$, such that

$$
h_{n}^{\prime}(z)=\prod_{k=1}^{n}\left(1-\overline{x_{k}} z\right)^{(3 \beta-2) t_{k}}
$$

where $\left|x_{k}\right|=1,0 \leqslant t_{k} \leqslant 1, \sum_{k=1}^{n} t_{k}=1$, which is dense in the family $\mathcal{G}(\beta)$.

Each fractional transformation $\Omega(z)=\Psi_{k}(z)=1 /\left(1-\overline{x_{k}} z\right)$ maps the unit disk $\mathbb{D}$ onto the half plane $\operatorname{Re}(\Omega)>0$, so that $\left|\arg \left(1-\overline{x_{k}} z\right)\right|<\pi / 2$. 
Now, we set $F=h+\epsilon g$, where $|\epsilon|=1$, hence

$$
\frac{z F^{\prime}(z)}{W(z)}=\frac{z\left(h^{\prime}(z)+\epsilon g^{\prime}(z)\right)}{W(z)}=z^{\frac{3-3 \beta}{2}} \prod_{k=1}^{n}\left(1-\overline{x_{k}} z\right)^{(3 \beta-2) t_{k}},
$$

where $\left|x_{k}\right|=1,0 \leqslant t_{k} \leqslant 1$ and $\sum_{k=1}^{n} t_{k}=1$. If we fix $\beta=1 / 3$ or $\beta=1$, then

$$
\begin{aligned}
\left|\arg \left(\frac{z F^{\prime}(z)}{W(z)}\right)\right| & \leqslant\left|\arg \left(z^{\frac{3-3 \beta}{2}}\right)+\sum_{k=1}^{n} \arg \left(1-\overline{x_{k}} z\right)^{(3 \beta-2) t_{k}}\right| \leqslant \\
& \leqslant \frac{3-3 \beta}{2}|\arg z|+(3 \beta-2) \sum_{k=1}^{n} t_{k}\left|\arg \left(1-\overline{x_{k}} z\right)\right|< \\
& <\frac{3-3 \beta}{2} \pi+\frac{(3 \beta-2)}{2} \pi \sum_{k=1}^{n} t_{k}=\frac{\pi}{2} .
\end{aligned}
$$

It follows that $\operatorname{Re}\left(\frac{z F^{\prime}(z)}{W(z)}\right)>0$, where $W(z)=z^{\frac{3 \beta-1}{2}}\left(1+\epsilon z^{n}\right)$ is starlike for each $|\epsilon|=1$. Thus, in view of Lemma A, tje function $f=h+\bar{g}$ is close-to-convex in $\mathbb{D}$.

Remark. Note that Theorem 1 provides a slight generalization of the result by Muhanna and Ponnusamy [12, Theorem 6]. For $\beta=1 / 3$ or $\beta=1$, let

$$
h(z)=\frac{1-(1-z)^{3 \beta-1}}{3 \beta-1} \quad \text { and } \quad g(z)=\lambda \int_{0}^{z} t^{n}(1-t)^{3 \beta-2} d t .
$$

Clearly, $h \in \mathcal{G}(\beta)$ and $f(z)=h+\bar{g}$ is locally univalent for $|\lambda| \leqslant 1$. Further, for $\beta=1 / 3$ or $\beta=1$, the function $W(z)=z^{\frac{3 \beta-1}{2}}\left(1+\epsilon \lambda z^{n}\right)$ is starlike for each $|\epsilon|=1$ and $|\lambda| \leqslant \frac{3 \beta-1}{3 \beta+2 n-1}$, as

$$
\left|\frac{z W^{\prime}(z)}{W(z)}-1\right|=\left|\frac{3 \beta-3}{2}+n \frac{\epsilon \lambda z^{n}}{1+\epsilon \lambda z^{n}}\right|<\frac{3-3 \beta}{2}+n \frac{|\lambda|}{1-|\lambda|} \leqslant 1,
$$

for $z \in \mathbb{D}$. Therefore, in view of Theorem 1 , the function

$$
f(z)=\frac{1-(1-z)^{3 \beta-1}}{3 \beta-1}+\overline{\int_{0}^{z} t^{n}(1-t)^{3 \beta-2} d t}
$$


for $\beta \in\{1 / 3,1\}, n \geqslant 1,|\lambda| \leqslant \frac{3 \beta-1}{3 \beta+2 n-1}, z \in \mathbb{D}$, is close-to-convex. In particular, the function

$$
f_{1}(z)=z-\frac{z^{2}}{2}+\overline{\int_{0}^{z} t^{n}(1-t) d t}, \quad n \geqslant 1,|\lambda| \leqslant \frac{1}{n+1}, z \in \mathbb{D}
$$

is close-to-convex. Images of the unit disk under the function $f_{1}$ for various values of $n$ and $\lambda$ are drawn in Figure 1(a)-Figure 1(f). Closer examination of Figure 1(c) and Figure 1(f) shows that the function $f_{1}$ is not univalent in $\mathbb{D}$ for certain values of $n$ and $\lambda$.
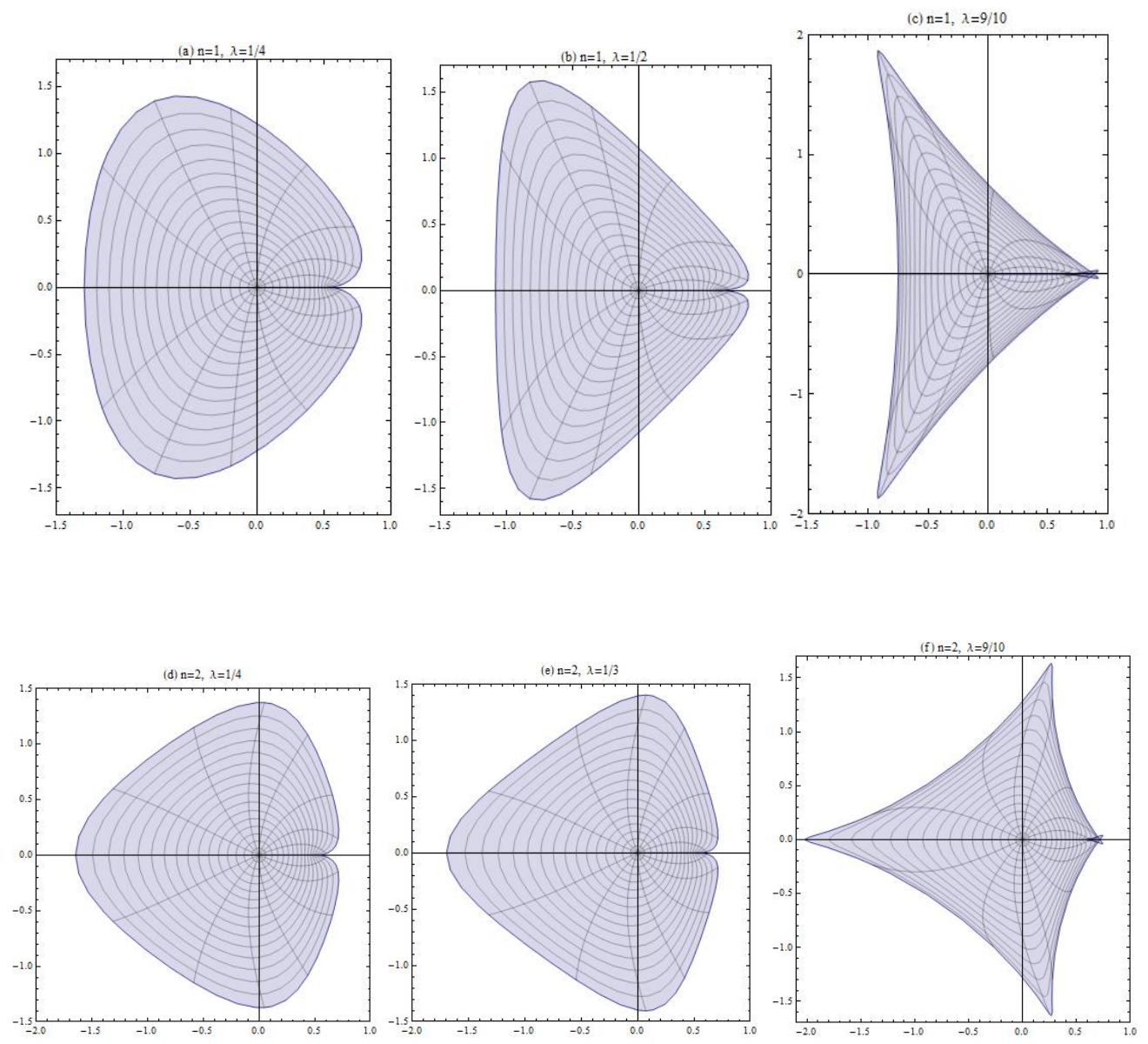

Figure 1 
The results in Theorem 2 and Theorem 3 provide bounds on the preSchwarzian norm $\left\|P_{f}\right\|$ and $\left\|\mathcal{P}_{f}\right\|$, respectively, for functions in the class $\mathcal{G}_{\mathcal{H}}(\alpha, \beta)$.

Theorem 2. Let $\alpha \in[0,1), \beta \in(2 / 3,1]$. If $f=h+\bar{g} \in G_{\mathcal{H}}(\alpha, \beta)$, then $\left\|P_{f}\right\| \leqslant 2(3 \beta-1)$. The bound is sharp.

Proof. If $f=h+\bar{g} \in G_{\mathcal{H}}(\alpha, \beta)$, then $h \in \mathcal{G}(\beta)$. Hence, we have

$$
1+\frac{z h^{\prime \prime}(z)}{h^{\prime}(z)} \prec \frac{1+(1-3 \beta) z}{1-z}=\psi(z), \quad z \in \mathbb{D},
$$

where $\psi$ is a starlike function in $\mathbb{D}$. Let $k \in \mathcal{A}$ be such that

$$
1+\frac{z k^{\prime \prime}(z)}{k^{\prime}(z)}=\frac{1+(1-3 \beta) z}{1-z}, \quad z \in \mathbb{D} .
$$

A computation gives $k^{\prime}(z)=(1-z)^{3 \beta-2}$. By Lemma B, we conclude that $h^{\prime}(z) \prec k^{\prime}(z)=(1-z)^{3 \beta-2}(z \in \mathbb{D})$, which implies

$$
h^{\prime}(z)=(1-\phi(z))^{3 \beta-2},
$$

for $\phi(z) \in \mathcal{B}_{0}$. Further, the dilation $w \in \mathcal{B}_{\alpha}$ satisfies the inequality

$$
\left|\frac{w(z)-w(0)}{1-\overline{w(0)} w(z)}\right| \leqslant|z|, \quad|z|=r<1,
$$

from which it follows that

$$
\left|w(z)-\frac{w(0)\left(1-r^{2}\right)}{1-|w(0)|^{2} r^{2}}\right| \leqslant \frac{r\left(1-|w(0)|^{2}\right)}{1-|w(0)|^{2} r^{2}} .
$$

Since $|w(0)|=\left|b_{1}\right|=\alpha$, we obtain

$$
1-|w(z)| \leqslant \frac{(1-\alpha)(1+r)}{1-\alpha r}, \quad|z|=r<1 .
$$

Now, using (2) and (8), we obtain

$$
\begin{aligned}
\left|P_{f}\right| & =\left|\frac{h^{\prime \prime}(z)}{h^{\prime}(z)}+\frac{2 q^{\prime}(z) q(z)}{1+|q(z)|^{2}}\right|=\left|\frac{h^{\prime \prime}(z)}{h^{\prime}(z)}\right|+\frac{\left|w^{\prime}(z)\right|}{1+|w(z)|} \leqslant \\
& \leqslant(3 \beta-2) \frac{1+|\phi(z)|}{1-|z|^{2}}+\frac{1-|w(z)|}{1-|z|^{2}} .
\end{aligned}
$$


Further, using (10) in the last inequality, we obtain

$$
\begin{aligned}
\left\|P_{f}\right\| & =\sup _{z \in \mathbb{D}}\left(1-|z|^{2}\right)\left|P_{f}\right| \leqslant \\
& \leqslant \sup _{0<|z|=r<1}\left[(3 \beta-2)(1+|\phi(z)|)+(1-\alpha) \frac{1+r}{1-\alpha r}\right] \leqslant \\
& \leqslant 2(3 \beta-2)+(1-\alpha) \sup _{0<r<1} X(r),
\end{aligned}
$$

where $X(r)=\frac{1+r}{1-\alpha r}$. Note that $X^{\prime}(r)=(1+\alpha) /(1-\alpha r)^{2}>0$, which shows that $X(r)$ is an increasing function of $r(0<r<1)$; hence

$$
\sup _{0<r<1} X(r)=X(1)=\frac{2}{1-\alpha} \text {. }
$$

Therefore, $\left\|P_{f}\right\| \leqslant 6 \beta-2$.

The last inequality becomes equality for the function $h \in \mathcal{G}(\beta)$, given by

$$
h(z)=\frac{1-(1-z)^{3 \beta-1}}{3 \beta-1}, \quad z \in \mathbb{D}, \beta \in(2 / 3,1],
$$

and $w$ is given by $w(z)=(z-\alpha) /(1-\alpha z), z \in \mathbb{D}, \alpha \in \mathbb{R},|\alpha|<1$. Indeed,

$$
\left\|P_{f}\right\|=\sup _{|z|=r<1}\left\{\frac{3 \beta-2}{|1-z|}+(1-\alpha) \frac{(1+r)}{(1-\alpha r)}\right\}=2(3 \beta-1)
$$

can be easily computed. This proves the result.

Theorem 3. Let $\alpha \in[0,1), \beta \in(2 / 3,1]$. If $f=h+\bar{g} \in G_{\mathcal{H}}(\alpha, \beta)$, then $\left\|\mathcal{P}_{f}\right\| \leqslant 3(2 \beta-1)$. The bound is sharp.

Proof. If $f=h+\bar{g} \in G_{\mathcal{H}}(\alpha, \beta)$, then $h \in \mathcal{G}(\beta)$. Following the proof of Theorem 2, we have $h^{\prime}(z)=(1-\phi(z))^{3 \beta-2}$ for $\phi \in \mathcal{B}_{0}$. Now, using the definition of pre-Schwarzian derivative $\mathcal{P}_{f}$ and (2), we have

$$
\begin{aligned}
\left|\mathcal{P}_{f}(z)\right| & =\left|\frac{h^{\prime \prime}(z)}{h^{\prime}(z)}-\frac{\overline{w(z)} w^{\prime}(z)}{1-|w(z)|^{2}}\right| \leqslant \\
& \leqslant(3 \beta-2) \frac{\left|\phi^{\prime}(z)\right|}{|1-\phi(z)|}+\frac{|w(z)|}{1-|z|^{2}} .
\end{aligned}
$$

The remaining proof follows similarly to the proof of Theorem 2 . 
Taking $\beta=1$ in Theorem 2 and Theorem 3, we obtain

Corollary. If $f=h+\bar{g} \in \mathcal{H}$, where $h \in \mathcal{G}$ and $w=g^{\prime}(z) / h^{\prime}(z) \in \mathcal{B}_{\alpha}$, then $\left\|P_{f}\right\| \leqslant 4$ and $\left\|\mathcal{P}_{f}\right\| \leqslant 3$. The bounds are sharp.

The following result provides a bound on the Bloch constant for functions in the class $G_{\mathcal{H}}(\alpha, \beta)$.

Theorem 4. Let $\alpha \in[0,1)$ and $\beta \in(2 / 3,1]$. If $f=h+\bar{g} \in G_{\mathcal{H}}(\alpha, \beta)$, then the upper bound on the Bloch constant $\mathcal{B}_{f}$ is given by

$$
\mathcal{B}_{f} \leqslant \frac{(1+\alpha)\left(1-r_{0}\right)\left(1+r_{0}\right)^{3 \beta}}{1+\alpha r_{0}}
$$

where $r_{0}$ is the unique root of the equation

$$
3 \alpha \beta r^{2}+(1+\alpha+3 \beta-3 \alpha \beta) r-3 \beta+\alpha+1=0
$$

in the interval $(0,1)$. The estimate of the Bloch constant is sharp.

Proof. Let $f=h+\bar{g} \in G_{\mathcal{H}}(\alpha, \beta)$; then $h \in \mathcal{G}(\beta)$. In view of (8), we have

$$
(1-r)^{3 \beta-2} \leqslant\left|h^{\prime}(z)\right| \leqslant(1+r)^{3 \beta-2} \quad(|z|=r<1) .
$$

Now, using (9) and (14), we have

$$
\begin{aligned}
\mathcal{B}_{f} & =\sup _{z \in \mathbb{D}}\left(1-|z|^{2}\right)\left(\left|h^{\prime}(z)\right|+\left|g^{\prime}(z)\right|\right) \leqslant \\
& \leqslant \sup _{0<r<1}\left(1-r^{2}\right)(1+r)^{(3 \beta-2)}\left(1+\frac{r+\alpha}{1+\alpha r}\right)= \\
& =(1+\alpha) \sup _{0<r<1} Q(r),
\end{aligned}
$$

where $Q(r):=\frac{(1-r)(1+r)^{3 \beta}}{1+\alpha r}$. A computation gives

$$
Q^{\prime}(r)=\frac{(1+r)^{3 \beta-1}}{(1+\alpha r)^{2}}\left\{3 \beta-\alpha-1-(1+\alpha+3 \beta-3 \alpha \beta) r-3 \alpha \beta r^{2}\right\} .
$$

Hence, $Q^{\prime}(r)=0$, if $S(r)=0$ for $r \in(0,1)$, where

$$
S(r)=3 \beta-\alpha-1-(1+\alpha+3 \beta-3 \alpha \beta) r-3 \alpha \beta r^{2} .
$$

Note that $S(0)=3 \beta-\alpha-1>0$ and $S(1)=-2(1+\alpha)<0$, so there exists a root $r_{0} \in(0,1)$, such that $S\left(r_{0}\right)=0$. Now, it is sufficient to prove 
that $r_{0}$ is unique. For this, it is sufficient to show that $S(r)$ is a decreasing function of $r$ for $0<r<1$. Note that,

$$
S^{\prime}(r)=-(1+\alpha+3 \beta(1-\alpha)+6 \alpha \beta r)<0,
$$

hence, $r_{0}$ is a unique root of $Q^{\prime}(r)=0$ for $0<r<1$. Thus,

$$
\sup _{0<r<1} Q(r)=\frac{\left(1-r_{0}\right)\left(1+r_{0}\right)^{3 \beta}}{1+\alpha r_{0}},
$$

where $r_{0}$ is a unique root of equation (13). The equality in (12) holds for the function $f=h+\bar{g} \in G_{\mathcal{H}}(\alpha, \beta)$, where $h$ is given by (11) and $w(z)=(z-\alpha) /(1-\alpha z), z \in \mathbb{D}, \alpha \in \mathbb{R},|\alpha|<1$.

The results in Theorem 5 and Theorem 6 given below provide the bounds on coefficients and Fekete-Szegö functional for the co-analytic part of functions in the class $G_{\mathcal{H}}(\alpha, \beta)$.

Theorem 5. If $f=h+\bar{g} \in G_{\mathcal{H}}(\alpha, \beta)$, where $h$ and $g$ are given by (1), then

$$
\left|b_{2}\right| \leqslant \frac{1-2 \alpha-\alpha^{2}+3 \alpha \beta}{2} \text {. }
$$

This estimate is sharp. Moreover,

$$
\left|b_{n}\right| \leqslant \frac{1-\alpha^{2}}{n}[1+(3 \beta-2)(1+\log (n-2))]+\frac{\alpha(3 \beta-2)}{n(n-1)}, \quad n=3,4, \ldots .
$$

Proof. Let $w \in \mathcal{B}_{\alpha}$, where $w(z)=\sum_{n=0}^{\infty} c_{n} z^{n}$ with $\left|c_{0}\right|=\alpha$. Using the series expansions (1) along with the relation $g^{\prime}(z)=w(z) h^{\prime}(z)$, we obtain

$$
n b_{n}=\sum_{p=0}^{n-1}(p+1) a_{p+1} c_{n-p-1}, \quad n=2,3, \ldots,
$$

For $n=2$, we have $2 b_{2}=a_{1} c_{1}+2 a_{2} c_{0}$. Using (2) and Lemma C(a), we get

$$
\begin{aligned}
2\left|b_{2}\right| & \leqslant\left|a_{1}\right|\left|c_{1}\right|+2\left|a_{2}\right|\left|c_{0}\right| \leqslant\left(1-\left|c_{0}\right|^{2}\right)+(3 \beta-2)\left|c_{0}\right| \leqslant \\
& \leqslant 1-2 \alpha-\alpha^{2}+3 \alpha \beta .
\end{aligned}
$$

To show the sharpness of (16), consider the harmonic mapping $f=h+\bar{g}$, where $h$ is given by (11) and the dilatation $w=g^{\prime} / h^{\prime}$ is given by

$$
w(z)=\frac{z-\alpha}{1-\alpha z}, \quad \alpha \in \mathbb{R},|\alpha|<1, z \in \mathbb{D} .
$$


Hence,

$$
\begin{aligned}
g^{\prime}(z)=w(z) h^{\prime}(z)=\frac{z-\alpha}{1-\bar{\alpha} z}(1-z)^{3 \beta-2} & = \\
=-\alpha & +\left(1-2 \alpha-\alpha^{2}+3 \alpha \beta\right) z+\ldots
\end{aligned}
$$

This shows the sharpness of (16).

Similarly, from (18) for $(n=3,4, \ldots)$, we obtain:

$$
\begin{aligned}
\left|b_{n}\right| & \leqslant \frac{1}{n} \sum_{p=0}^{n-1}(p+1)\left|a_{p+1}\right|\left|c_{n-p-1}\right|= \\
& =\frac{1}{n}\left[\left|a_{1}\right|\left|c_{n-1}\right|+\sum_{p=1}^{n-2}(p+1)\left|a_{p+1}\right|\left|c_{n-p-1}\right|+n\left|a_{n}\right|\left|c_{0}\right|\right]= \\
& =\frac{1}{n}\left|c_{n-1}\right|+\frac{1}{n} \sum_{p=1}^{n-2}(p+1)\left|a_{p+1}\right|\left|c_{n-p-1}\right|+\left|a_{n}\right|\left|c_{0}\right| .
\end{aligned}
$$

So, we have $\left|c_{n-p-1}\right| \leqslant 1-\left|c_{0}\right|^{2}=1-\alpha^{2}$. Hence, using Lemma C(a), we get

$$
\begin{aligned}
\left|b_{n}\right| & \leqslant \frac{1-\alpha^{2}}{n}+\frac{\left(1-\alpha^{2}\right)(3 \beta-2)}{n} \sum_{p=1}^{n-2} \frac{1}{p}+\frac{\alpha(3 \beta-2)}{n(n-1)}= \\
& =\frac{1-\alpha^{2}}{n}[1+(3 \beta-2)(1+\log (n-2))]+\frac{\alpha(3 \beta-2)}{n(n-1)} .
\end{aligned}
$$

This completes the proof of the theorem.

Theorem 6. Let $f=h+\bar{g} \in G_{\mathcal{H}}(\alpha, \beta)$, where $h$ and $g$ are given by (1). Then for $\mu \in \mathbb{R}$, we have

$$
\begin{aligned}
&\left|b_{3}-\mu b_{2}^{2}\right| \leqslant \frac{1-\alpha^{2}}{12}\{\left.4(3 \beta-1)+3|\mu|\left(1-\alpha^{2}-4 \alpha+6 \alpha \beta\right)\right\}+ \\
&+\alpha \max \left\{\frac{3 \beta-2}{2}\left|1-\beta-\frac{(3 \beta-2) \mu \alpha}{2}\right|, \frac{3 \beta-2}{6}\right\}
\end{aligned}
$$

and

$$
\left|b_{n+1}-b_{n}\right| \leqslant \frac{2 \alpha(3 \beta-2)}{n^{2}-1}+\frac{1-\alpha^{2}}{n+1}\{1+(3 \beta-2)(1+\log (n-1))\}+
$$




$$
+\frac{1-\alpha^{2}}{n}\{1+(3 \beta-2)(1+\log (n-2))\} .
$$

Proof. From (18), we have

$$
b_{2}=\frac{1}{2} c_{1}+a_{2} c_{0} \quad \text { and } \quad b_{3}=\frac{1}{3} c_{2}+\frac{2}{3} a_{2} c_{1}+a_{3} c_{0} .
$$

Now,

$$
\begin{aligned}
\left|b_{3}-\mu b_{2}^{2}\right|=\left|\frac{1}{3} c_{2}+\frac{2}{3} a_{2} c_{1}+a_{3} c_{0}-\mu\left(\frac{1}{2} c_{1}+a_{2} c_{0}\right)^{2}\right| & \leqslant \\
& \leqslant \frac{1}{3}\left|c_{2}\right|+\frac{1}{4}|\mu|\left|c_{1}\right|^{2}+\frac{2}{3}\left|a_{2}\right|\left|c_{1}\right|+|\mu|\left|a_{2}\right|\left|c_{1}\right|\left|c_{0}\right|+\left|c_{0}\right|\left|\left(a_{3}-\mu c_{0} a_{2}^{2}\right)\right| .
\end{aligned}
$$

Using Lemma C, we obtain

$$
\begin{array}{r}
\left|b_{3}-\mu b_{2}^{2}\right| \leqslant \frac{1}{3}\left(1-\alpha^{2}\right)+\frac{1}{4}|\mu|\left(1-\alpha^{2}\right)^{2}+\frac{1}{3}(3 \beta-2)\left(1-\alpha^{2}\right)+ \\
+\frac{1}{2} \alpha(3 \beta-2)|\mu|\left(1-\alpha^{2}\right)+\alpha\left|a_{3}-\mu \alpha a_{2}^{2}\right| \leqslant \\
\leqslant \frac{1}{3}\left(1-\alpha^{2}\right)(3 \beta-1)+\frac{1}{4}|\mu|\left(1-\alpha^{2}\right)\left\{1-\alpha^{2}-4 \alpha+6 \alpha \beta\right\}+ \\
+\alpha \max \left\{\frac{3 \beta-2}{2}\left|1-\beta-\frac{(3 \beta-2) \mu \alpha}{2}\right|, \frac{3 \beta-2}{6}\right\} .
\end{array}
$$

Further, using (18), we get

$$
\begin{gathered}
\left|b_{n+1}-b_{n}\right|=\left|\frac{1}{n+1} \sum_{p=1}^{n+1} p a_{p} c_{n-p+1}-\frac{1}{n} \sum_{p=1}^{n} p a_{p} c_{n-p}\right|= \\
=\left|\frac{1}{n+1} \sum_{p=1}^{n} p a_{p} c_{n-p+1}+a_{n+1} c_{0}-\frac{1}{n} \sum_{p=1}^{n-1} p a_{p} c_{n-p}-a_{n} c_{0}\right| \leqslant \\
\leqslant\left|a_{n+1}\right|\left|c_{0}\right|+\left|a_{n}\right|\left|c_{0}\right|+\frac{1}{n+1} \sum_{p=1}^{n} p\left|a_{p}\right|\left|c_{n-p+1}\right|+\frac{1}{n} \sum_{p=1}^{n-1} p\left|a_{p}\right|\left|c_{n-p}\right|= \\
=\left|a_{n+1}\right|\left|c_{0}\right|+\left|a_{n}\right|\left|c_{0}\right|+\frac{\left|a_{1}\right|\left|c_{n}\right|}{n+1}+\frac{1}{n+1} \sum_{p=2}^{n} p\left|a_{p}\right|\left|c_{n-p+1}\right|+ \\
+\frac{\left|a_{1}\right|\left|c_{n-1}\right|}{n}+\frac{1}{n} \sum_{p=2}^{n-1} p\left|a_{p}\right|\left|c_{n-p}\right| \leqslant
\end{gathered}
$$




$$
\begin{gathered}
\leqslant \frac{\alpha(3 \beta-2)}{n(n+1)}+\frac{\alpha(3 \beta-2)}{n(n-1)}+\frac{1-\alpha^{2}}{n+1}+\frac{\left(1-\alpha^{2}\right)}{n+1}(3 \beta-2) \sum_{p=2}^{n} \frac{1}{p-1}+ \\
+\frac{1-\alpha^{2}}{n}+\frac{\left(1-\alpha^{2}\right)}{n}(3 \beta-2) \sum_{p=2}^{n-1} \frac{1}{p} \leqslant \\
\leqslant \frac{2 \alpha(3 \beta-2)}{\left(n^{2}-1\right)}+\frac{1-\alpha^{2}}{n+1}+\frac{\left(1-\alpha^{2}\right)}{n+1}(3 \beta-2) \sum_{p=1}^{n-1} \frac{1}{p}+\frac{1-\alpha^{2}}{n}+ \\
+\frac{\left(1-\alpha^{2}\right)}{n}(3 \beta-2) \sum_{p=1}^{n-2} \frac{1}{p-1} \leqslant \\
\leqslant \frac{2 \alpha(3 \beta-2)}{n^{2}-1}+\frac{1-\alpha^{2}}{n+1}+\frac{\left(1-\alpha^{2}\right)}{n+1}(3 \beta-2)[1+\log (n-1)]+\frac{1-\alpha^{2}}{n}+ \\
+\frac{\left(1-\alpha^{2}\right)}{n}(3 \beta-2)[1+\log (n-2)]= \\
=\frac{2 \alpha(3 \beta-2)}{n^{2}-1}+\frac{1-\alpha^{2}}{n+1}[1+(3 \beta-2)(1+\log (n-1))]+ \\
+\frac{1-\alpha^{2}}{n}[1+(3 \beta-2)(1+\log (n-2))]
\end{gathered}
$$

This completes the proof of theorem.

Finally, we establish the following result containing inequalities on the co-analytic part of functions in class $G_{\mathcal{H}}(\alpha, \beta)$.

Theorem 7. Let $f=h+\bar{g} \in G_{\mathcal{H}}(\alpha, \beta)$, where $h$ and $g$ are given by (1). Then, for $|z|=r<1$, the following statements hold:

$$
\frac{(3 \beta-2) r}{1+r}-\frac{r\left(1-\alpha^{2}\right)}{(1-\alpha r)|r-\alpha|} \leqslant\left|\frac{z g^{\prime \prime}(z)}{g^{\prime}(z)}\right| \leqslant \frac{(3 \beta-2) r}{1-r}+\frac{r\left(1-\alpha^{2}\right)}{(1-\alpha r)|r-\alpha|}
$$

and

$$
\operatorname{Re}\left(1+\frac{z g^{\prime \prime}(z)}{g^{\prime}(z)}\right)<\frac{3 \beta}{2}+\frac{r\left(1-\alpha^{2}\right)}{(1-\alpha r)|r-\alpha|} .
$$

Proof. Let $f=h+\bar{g} \in G_{\mathcal{H}}(\alpha, \beta)$; then $g^{\prime}(z)=w(z) h^{\prime}(z)$. Now, taking the logarithmic derivative, we have

$$
\frac{z g^{\prime \prime}(z)}{g^{\prime}(z)}=\frac{z w^{\prime}(z)}{w(z)}+\frac{z h^{\prime \prime}(z)}{h^{\prime}(z)}
$$


In view of (10) and (2), we get

$$
\frac{|r-\alpha|}{1-\alpha r} \leqslant|w(z)| \leqslant \frac{r+\alpha}{1+\alpha r} \quad \text { and } \quad\left|w^{\prime}(z)\right| \leqslant \frac{1-\alpha^{2}}{(1-\alpha r)^{2}} .
$$

Further, using (8), we have

$$
\frac{z h^{\prime \prime}(z)}{h^{\prime}(z)}=-\frac{(3 \beta-2) \phi(z)}{1-\phi(z)}
$$

which gives the estimate

$$
\left|\frac{z h^{\prime \prime}(z)}{h^{\prime}(z)}\right| \leqslant \frac{(3 \beta-2) r}{1-r}, \quad|z|=r<1
$$

(use the condition $|\phi(z)| \leqslant 1$ ). Now, using (19)-(21), we obtain

$$
\left|\frac{z g^{\prime \prime}(z)}{g^{\prime}(z)}\right| \leqslant\left|\frac{z h^{\prime \prime}(z)}{h^{\prime}(z)}\right|+\left|\frac{z w^{\prime}(z)}{w(z)}\right| \leqslant \frac{(3 \beta-2) r}{1-r}+\frac{r\left(1-\alpha^{2}\right)}{(1-\alpha r)|r-\alpha|} .
$$

Similarly,

$$
\left|\frac{z g^{\prime \prime}(z)}{g^{\prime}(z)}\right| \geqslant\left|\frac{z h^{\prime \prime}(z)}{h^{\prime}(z)}\right|-\left|\frac{z w^{\prime}(z)}{w(z)}\right| \geqslant \frac{(3 \beta-2) r}{1+r}-\frac{r\left(1-\alpha^{2}\right)}{(1-\alpha r)|r-\alpha|} .
$$

Further, using the relation (20), we get

$$
\operatorname{Re}\left(\frac{z w^{\prime}(z)}{w(z)}\right) \leqslant\left|\frac{z w^{\prime}(z)}{w(z)}\right| \leqslant \frac{r\left(1-\alpha^{2}\right)}{(1-\alpha r)|r-\alpha|} .
$$

Hence,

$$
\begin{aligned}
\operatorname{Re}\left(1+\frac{z g^{\prime \prime}(z)}{g^{\prime}(z)}\right)=\operatorname{Re}\left(1+\frac{z h^{\prime \prime}(z)}{h^{\prime}(z)}\right)+\operatorname{Re} & \left(\frac{z w^{\prime}(z)}{w(z)}\right)< \\
& <\frac{3 \beta}{2}+\frac{r\left(1-\alpha^{2}\right)}{(1-\alpha r)|r-\alpha|} .
\end{aligned}
$$

This completes the proof.

Acknowledgement. The authors express their sincere thanks to the editors and referees for their valuable suggestions to improve the manuscript. 


\section{References}

[1] Avkhadiev F. G., Wirths K. J. Schwarz-Pick Type Inequalities. Birkhauser Verlag AG, Basel-Boston-Berlin, 2009.

DOI: http://10.1007/978-3-0346-0000-2

[2] Becker J. Löwnersche differentialgleichung und quasikonform fortsetzbare schlichte funktionen. J. Reine Angew. Math., 1972, vol. 255, pp. 23-43.

[3] Chen H., Gauthier P. M., Hengartner W. Bloch constants theorem for planar harmonic mappings. Proc. Amer. Math. Soc., 2000, vol. 128, no. 11, pp. 3231 -3240. DOI: http://10.1090/S0002-9939-00-05590-8

[4] Chen Sh., Ponnusamy S., Wang X., Bloch constant and Landau's theorem for planar p-harmonic mappings, J. Math. Anal. Appl., 2011, vol. 373, no. 1, pp. 102-110. DOI: http://10.1016/j.jmaa.2010.06.025

[5] Chuaqui M., Duren P., Osgood B. Curvature properties of planar harmonic mappings. Comput. Methods Funct. Theory, 2004, vol. 4, no. 4, pp. $127-142$. DOI: http://10.1007/BF03321060

[6] Clunie J. G., Sheil-Small T. Harmonic univalent functions. Ann. Acad. Sci. Fenn. Ser. AI., 1984, vol. 9, pp. 3-25.

DOI: http://10.5186/aasfm.1984.0905

[7] Colonna F. The Bloch constant of bounded harmonic mappings. Indiana Univ. Math. J., 1989, vol. 38, no. 4, pp. 829-840.

DOI: www.jstor.org/stable/24895369

[8] Hernández R., Martín M. Pre-Schwarzian and Schwarzian derivatives of harmonic mappings. J. Geom. Anal., 2015, vol. 25, pp. 64-91.

DOI: http://10.1007/s12220-013-9413-x

[9] Liu M.-S. Estimates on Bloch constants for planar harmonic mappings. Sci. China Ser. A, 2009, vol. 52, pp. 87-93.

DOI: http://10.1007/s11425-008-0090-3

[10] Liu M.-S., Luo L.-F., Luo X. Landau-Bloch type theorems for strongly bounded harmonic mappings. Monatsh. Math., 2020, vol. 191, pp. $175-185$.

DOI: https://doi.org/10.1007/s00605-019-01284-8

[11] Ma W., Minda D., A unified treatment of some special classes of univalent functions, in: Li Z., Ren F., Yang L., Zhang S., Proceedings of the Conference on Complex Analysis, International Press Inc., 1992, pp. 157-169.

[12] Muhanna Y. A., Ponnusamy S. Extreme points method and univalent harmonic mappings, In Complex Analysis and Dynamical Systems VI., Contemp. Math., 2016, vol. 667, pp. 223-237.

DOI: http://10.1090/conm/667 
[13] Obradović M., Ponnusamy S., Wirths K. J. Coefficient characterizations and sections for some univalent functions. Sib. Math. J., 2013, vol. 54, no. 4, pp. 679-696. DOI: http://10.1134/S0037446613040095

[14] Ozaki S. On the theory of multivalent functions. II. Sci. Rep. Tokyo Bunrika Daigaku. Sect. A., 1941, vol. 4, pp.45-87.

DOI: https://www.jstor.org/stable/43700364

[15] Ponnusamy S., Qiao J., Wang X. Uniformly locally univalent harmonic mappings. Proc. Indian Acad. Sci. (Math. Sci.), 2018, vol. 128, Article ID 0032. DOI: http://10.1007/s12044-018-0405-4

[16] Ponnusamy S., Prajapat J. K., Kaliraj A. S. Uniformly starlike and uniformly convex harmonic mappings.., J. Anal., 2015, vol. 23, pp. 121-129.

[17] Ponnusamy S., Starkov V. V. The Jacobian Conjecture and Injectivity Conditions., Bull. Malays. Math. Sci. Soc., 2018, vol. 41, pp. 2099-2115.

DOI: http://10.1007/s40840-018-0626-9

[18] Ponnusamy S., Vasudevarao A. Region of Variability of two subclasses of univalent functions. J. Math. Anal. Appl., 2007, vol. 332, no. 2, pp. 1322 -1333. DOI: http://10.1016/j.jmaa.2006.11.019

[19] Prajapat J. K., Manivannan M., Maharana S. Harmonic mappings with analytic part convex in one direction. J. Anal., 2020, vol. 28, pp. 261-272. DOI: http://10.1007/s41478-020-00226-0

[20] Umezava T. Analytic functions convex in one direction. J. Math. Soc. Japan, 1952, vol. 4, pp. 194-202. DOI: http://10.2969/jms j/00420194

[21] Yamashita S. Almost locally univalent functions. Monatsh Math., 1976, vol. 81, pp. $235-240$. DOI: http://10.1007/BF01303197

Received July 29, 2020.

In revised form, December 16, 2020.

Accepted December 17, 2020.

Published online February 15, 2021.

Department of Mathematics, Central University of Rajasthan,

Bandarsindri, Kishangarh-305817, Dist.-Ajmer, Rajasthan, India.

E-mail: jkprajapat@curaj.ac.in 